Rev. Biol. Trop., 47(3): 351-358, 1999

www.ucr.ac.cr www.ots.ac.cr www.ots.duke.edu

\title{
Hábitat y distribución de cinco especies de Quercus (Fagaceae) en la Meseta Central de Chiapas, México
}

\author{
J. G. Alvarez-Moctezuma ${ }^{1}$, S. Ochoa-Gaona ${ }^{2}$, B. H. J. de Jong² y M. L. Soto-Pinto ${ }^{2}$
}

1 Escuela de Biología. Universidad de Ciencias y Artes del Estado de Chiapas. A.P. 57, C.P. 29000. Tuxtla Gutiérrez, Chiapas, México. Tel: (961) 2-14-37. Fax: (961) 2-17-81. Correo electrónico: jogualmo@ chis1.telmex.net.mx

2 El Colegio de la Frontera Sur. A.P. 63, C.P. 29290. San Cristóbal de Las Casas, Chiapas, México. Tel: (967) 8-18-83. Fax: 5550 0164. Correo electrónico: sochoa@sclc.ecosur.mx

* Toda correspondencia debe dirigirse a este autor.

Recibido 6-V-1998. Corregido 24-II-1998. Aceptado 26-I-1998.

\begin{abstract}
We describe the habitat of species within the Fagaceae subgenus Lepidobalanus (genus Quercus) and identify environmental variables related to their distribution in the Meseta Central of Chiapas, Southern Mexico. In 258 plots a dominance index was used, combining tree density and crown cover, for Quercus peduncularis, $Q$. polymorpha, $Q$. rugosa, $Q$. sebifera and $Q$. segoviensis. The following variables were measured: altitude, precipitation from November through April (PPNA), exposure, slope, fuel-wood harvesting and grazing. Detrended Canonical Correspondence Analysis was used for the statistical evaluation of data. Negative relationships among the dominance index of $Q$. peduncularis and $Q$. segoviensis, and altitude and PPNA values were found. The dominance index of $Q$. rugosa and $Q$. segoviensis was correlated with exposure and intensity of fuel-wood harvesting. Altitude, and to a lesser extent PPNA, determined the distribution of the evaluated species. The results suggest that Quercus peduncularis, $Q$. polymorpha, $Q$. rugosa, $Q$. segoviensis and $Q$. sebifera display different ecological patterns.
\end{abstract}

Key words: Quercus, oak, habitat, altitude, precipitation, population dynamics, multivariate analysis.

En la Meseta Central de Chiapas dominan los bosques templados (Breedlove 1981). La mayor parte de los componentes del dosel de estos bosques son de afinidad holártica, siendo dominantes las especies de Pinus y Quercus (Quintana-Ascencio y González-Espinosa 1993). Las especies de encino (Quercus) son utilizadas en actividades forestales, artesanales, agrícolas y pecuarias de la región. Además por su alta calidad calorífica son la fuente principai de leña, de la cual depende la mayoría de los pobladores de la región (Berlín et al. 1974, Díaz Hernández et al. 1988, Breedlove y Laughlin 1993). Debido al intenso aprovecha- miento del que son objeto y al creciente cambio de uso del suelo, las poblaciones de Quercus están siendo fuertemente afectadas (Ramírez Marcial et al. 1996), ya que se les impide llegar a su madurez reproductiva, restringiendo su regeneración sexual (González-Espinosa et al. 1997), de tal manera que alrededor de los asentamientos humanos, las poblaciones de Quercus están severamente constreñidas (Collier 1975).

Hasta ahora el aprovechamiento de los encinos se ha realizado como una actividad básicamente extractiva (Montoya Gómez 1995), por lo que se hace necesario el establecimiento 
de programas de manejo y restauración que, por un lado, mantengan el recurso y por otro permitan el abasto a los habitantes de la región (Buschbacher 1990). La definición del hábitat de las especies, sienta bases para establecer programas de restauración, reforestación y manejo de los bosques, proponiendo para cada área la especie de Quercus más adecuada, aprovechando la adaptación de las especies nativas.

El objetivo de este trabajo fue el de caracterizar el hábitat e identificar las variables ambientales más importantes que definen la distribución de las especies del subgénero Lepidobalanus, género Quercus en la Meseta Central de Chiapas.

\section{MATERIALES Y MÉTODOS}

El trabajo se realizó en la Meseta Central de Chiapas, México (Fig. 1), la cual se extiende del noroeste al sureste, siendo su longitud de $160 \mathrm{~km}$ y su mayor anchura de 75 km, ocupando una superficie aproximada de 11000 $\mathrm{km}^{2}$. Ocupa altitudes entre los 1000 y 2500 $\mathrm{m}$, aunque pequeñas porciones se encuentran desde los 500 hasta los $2850 \mathrm{~m}$. La topografía es accidentada, predominando las rocas sedimentarias y en pequeñas porciones las ígneas (Müllerried 1957). Se distinguen los siguientes climas a) subcálido, b) subtemplado, c) subtemplado húmedo y d) templado (Cuadro 1, García 1987). Los tipos de vegetación más frecuentes son: bosque de Pinus $(11.9 \%$ de la superficie total), bosque de Pinus-Quercus (20.6\%), bosque de Quercus $(7.4 \%)$ y bosque mesófilo de montaña (3.6\%) (González-Espinosa et al. 1995).

El área de estudio se estratificó en función de los cuatro tipos climáticos, trazándose once transectos. Con una separación de 500 a 1000 metros, se establecieron 258 parcelas de 400 $\mathrm{m}^{2}(20 \times 20 \mathrm{~m})$. Veinte parcelas quedaron ubicadas en el clima subcálido, 151 en el subtemplado húmedo, siete en el subtemplado muy húmedo y 80 en el clima en el templado (Fig. 1).

Se registraron los valores de altitud, precipitación de noviembre a abril (PPNA, que en la zona de estudio corresponde a los meses de sequía), exposición, pendiente, intensidad de extracción de leña y pastoreo en cada una de las parcelas. Se midió la abundancia y cobertura de las especies del subgénero Lepidobalanus (género Quercus) y con base en estos datos se aplicó un índice de dominancia (ID) para las especies (Cuadro 2). Se consideraron únicamente a los individuos $\geq 1.30 \mathrm{~m}$ de altura. Debido a que el índice de cantidad de especies de Braun-Blanquet (1979) está diseñado para el estudio de comunidades, algunas de las categorías no se aplican para evaluar poblaciones de especies arbóreas en particular, por lo que adecuamos éste, conforme las necesidades del presente estudio, al cual denominamos ID. La utilización del índice de dominancia modificado presentó las siguientes ventajas: a) es una técnica rápida que permitió el estudio de una gran área en poco tiempo, b) es una técnica cualitativa, que no requirió mediciones (que tanto incomodan a los habitantes de esta conflictiva región), c) es una técnica barata que no requiere de equipo sofisticado y d) permite incluir en una variable criterios de abundancia y cobertura (Cuadro 2).

La altitud (m) fue calculada utilizando una carta topográfica (Anónimo 1974). La PPNA (mm) se obtuvo sumando la precipitación de noviembre a abril, utilizando la carta de efectos climáticos regionales (Anónimo 1984). La pendiente fue medida en campo con clinómetro (en grados). La exposición se obtuvo en campo mediante brújula (en grados), la cual fue transformada a una escala de cero a uno. El cero se asignó a las parcelas expuestas al sur y el uno a las expuestas al norte, estas últimas tienden a ser más húmedas. La fórmula utilizada para calcular la exposición fue la siguiente (Roberts 1986):

$$
\text { donde: } \begin{aligned}
& \mathrm{e}_{\mathrm{t}}=\left(\cos \left(\mathrm{e}_{\mathrm{o}}-30^{\circ}\right)+1\right) / 2 \\
& \mathrm{e}_{\mathrm{t}} \text { : exposición transformada } \\
& \mathrm{e}_{\mathrm{o}} \text { : exposición observada }
\end{aligned}
$$

Para las variables pastoreo y extracción de leña se utilizó una escala cualitativa de intensidad: 

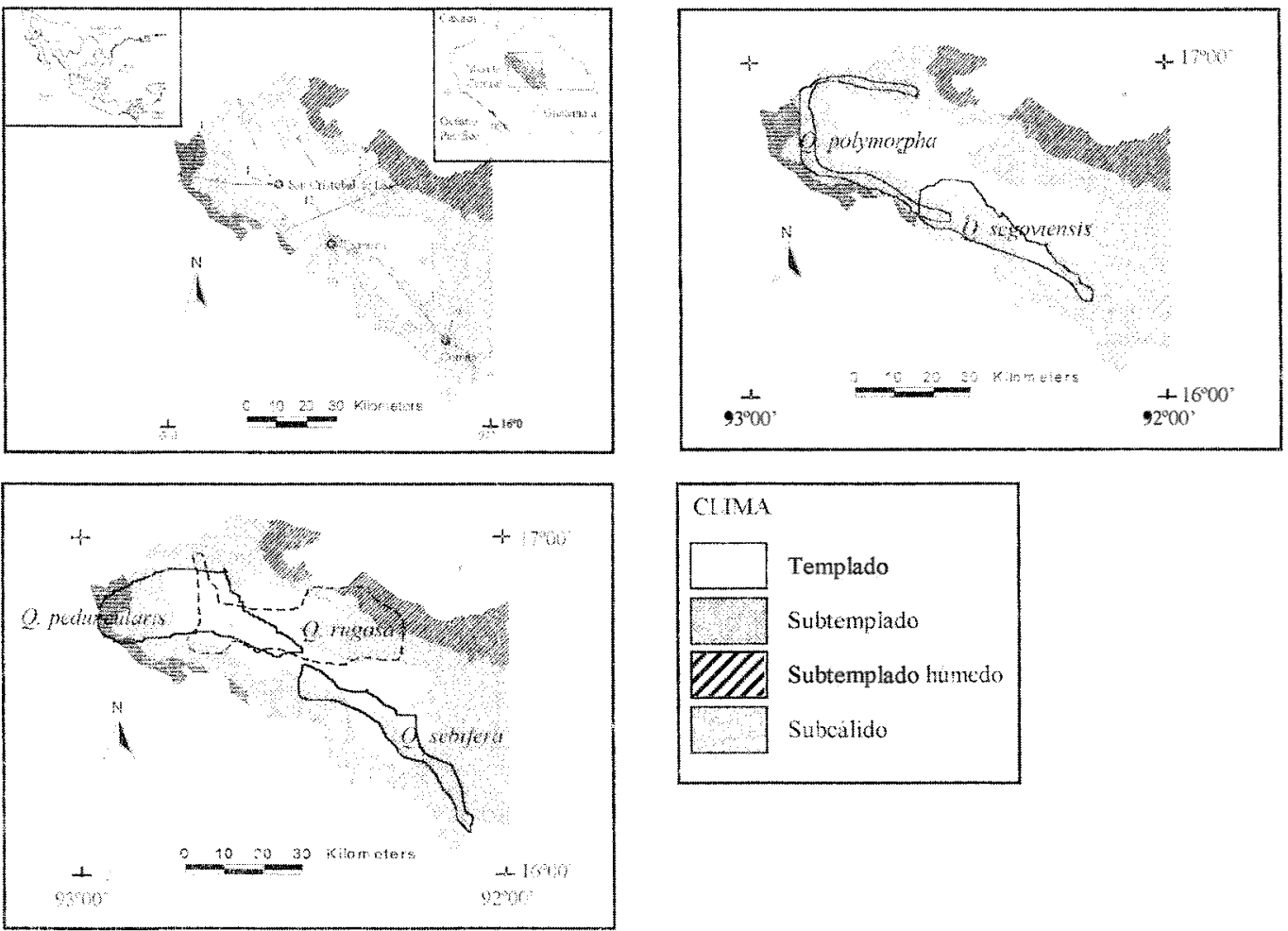

Fig. 1. Localización del área de estudio y distribución de las especies. A. Ubicación del estado Chiapas en la República Mexicana al margen superior izquierdo; localización de La Meseta Central de Chiapas y área de estudio al margen superior derecho y distribución de climas y localización de los once transectos de muestreo al centro. B. 2. Distribución de Quercus peduncular s y $Q$. polymorpha C. Distribución de $Q$. rugosa, $Q$. sebifera y $Q$. segoviensis en la Meseta Central de Chiapas. El polígono delimita la distribución de cada especie.

\section{CUADRO 1}

Características de los climas presentes en el área de estudio (García 1987)

\begin{tabular}{lllll} 
& Subcálido & Subtemplado & $\begin{array}{l}\text { Subtemplado } \\
\text { húmedo }\end{array}$ & \multicolumn{1}{c}{ Templado } \\
Temp. Media Anual $\left({ }^{\circ} \mathrm{C}\right)$ & 18 a 22 & $>18$ & $>18$ & 12 a 18 \\
Temp. mes más frío $\left({ }^{\circ} \mathrm{C}\right)$ & $>18$ & -3 a 18 & -3 y 18 & -3 a 18 \\
Temp. mes más caliente $\left({ }^{\circ} \mathrm{C}\right)$ & $>20$ & 6.5 a 22 & 6.5 a 22 & 6.5 a 22 \\
Oscilación térmica & Baja & Baja & Baja & Baja \\
Mes más caliente del año $\left({ }^{\circ} \mathrm{C}\right)$ & Antes de julio & Antes de julio & Antes de julio & Antes de julio \\
Período más lìuvioso & Mayo a sept. & Mayo a sept. & Todo el año & Mayo a sept. \\
Lluvias de nov.-abril $(\%)$ & 1 & $<5$ & Hasta 18 & $<5$ \\
Período de sequía intraestival & Agosto & Agosto & Agosto & Agosto \\
Altitud (m.s.n.m.) & 800 a 1200 & 1200 a 2000 & 1200 a 1800 & 2000 a 2500
\end{tabular}

ausencia (cero), baja (uno). media (dos) y alta (tres).

Se elaboraron mapas de distribución de las especies utilizando los datos de campo y la in- formación obtenida de los herbarios ECO-SCH, CHIP, MEXU, ENCB E INIFA. Los puntos extremos registrados fueron unidos formando un polígono (Fig. 1). Se recolectaron ejemplares 
CUADRO 2

Índice de Dominancia (ID) y criterios utilizados para su definición

$\begin{array}{ccc}\text { ID } & \text { Cobertura }(\%) & \begin{array}{c}\text { Número de } \\ \text { individuos } \\ \text { / parcela }\end{array} \\ 1 & <10 & 1-2 \\ 2 & 10-25 & 1-2 \\ 3 & <25 & 3-10 \\ 4 & <10 & >10 \\ 5 & 10-25 & >10 \\ 6 & 25-50 & \text { Cualquier número } \\ 7 & 50-75 & \text { Cualquier número } \\ 8 & >75 & \text { Cualquier número }\end{array}$

de individuos con estructuras reproductoras. La determinación se hizo mediante el corrimiento de claves botánicas y cotejo con ejemplares de herbario. Se confirmó la identificación con un especialista. Los ejemplares quedaron depositados en los herbarios ECO-SCH, FCME y UNICACH.

Para el análisis estadístico, las parcelas fueron consideradas como unidades independientes. Sólo se incluyeron las parcelas en las cuales las especies tenían un ID $\geq 3$. Lo anterior se basó en la consideración de que el índice de dominancia utilizado (al igual que el de BraunBlanquet) tiene la desventaja de sobrestimar las parcelas con uno o dos individuos de baja cobertura, lo que influye sobre el análisis estadístico (Cuadro 2). En los sitios donde se encontraron dos o tres especies del subgénero $L e$ pidobalanus; generalmente sólo una de ellas presentaba valores de ID $\geq 3$. La relación entre cada una de las especies con cada variable ambiental fue examinada aplicando el coeficiente de correlación. Se utilizó el Detrended Canonical Correspondence Analysis (DCCA, Hill y Gauch 1980, Ter Braak 1988). El DCCA combina la información ambiental con los datos de las especies dentro de un mismo análisis. El uso de los análisis multivariados permite interpretar de manera rápida un conjunto complejo de datos, mediante la generación de variables teóricas (los ejes de ordenación) los cuales representan clines en la estructura de la comunidad. La posición de cada especie en las gráfi- cas de ordenación, proporciona una representación visual de su respuesta al ambiente (Vickery et al. 1997). En el diagrama de dos ejes, la orientación y magnitud de cada variable ambiental con relación a los ejes, está representada por una flecha y señala la importancia de la variable en la distribución de las especies. El nivel de significancia entre las variables ambientales y el primer gradiente de cambio en el ID $\geq 3$ de las especies fueron calculados utilizando la prueba de permutación de Monte Carlo (Ter Braak 1988).

\section{RESULTADOS}

Quercus peduncularis se encontró en $38 \%$ de las parcelas estudiadas; $Q$. sebifera en $32 \%$; Q. rugosa en $27 \%$; Q. segoviensis en $25 \%$ y $Q$. polymorpha en $12 \%$.

La altitud estuvo correlacionada en forma negativa con los valores de ID $\geq 3$ de Quercus peduncularis y $Q$. segoviensis $(\mathrm{p}<0.001$ y $\mathrm{p}<0.05)$. La PPNA estuvo negativamente correlacionada con los valores de los ID $\geq 3$ de Quercus peduncularis y $Q$. segoviensis $(\mathrm{p}<0.001$ y $\mathrm{p}<0.05)$ y positivamente correlacionada con $Q$. polymorpha $(\mathrm{p}<0.05)$. Los valores más altos de los ID para las primeras dos especies se encontraron en las parcelas de menor PPNA, mientras que las parcelas donde se encontró $Q$. polymorpha generalmente fueron las de mayor cantidad de lluvias en invierno. La exposición estuvo positivamente correlacionada con Quercus rugosa y en forma negativa con $Q$. segoviensis $(\mathrm{p}<0.05)$. La primera especie se encontró mejor representada en las parcelas con exposición norte, o sea la de mayor humedad, en cambio la segunda especie en las parcelas con exposición sur. $\mathrm{La}$ pendiente no mostró correlación estadísticamente significativa con ninguna especie. $\mathrm{La}$ extracción de leña mostró correlación negativa con Quercus rugosa y $Q$. segoviensis $(\mathrm{p}<0.01$ y $\mathrm{p}<0.05)$. Estas dos especies están más afectadas por las actividades humanas que involucran la extracción de leña. El pastoreo no fue estadísticamente significativo 


\section{CUADRO 3}

Matriz de correlación de los ID $\geq 3$ de las especies de Quercus (Lepidobalanus) con las variables evaluadas. 1: Q. peduncularis, 2: Q. polymorpha, 3: Q. rugosa, 4: Q. sebifera y 5: Q. segoviensis

$\begin{array}{lccccc} & 1 & 2 & 3 & 4 & 5 \\ \text { Altitud } & -0.80 * * * & -0.04 & +0.01 & -0.01 & -0.23 * \\ \text { Precipitación nov-abril (PPNA) } & -0.42 * * * & +0.21 * & -0.12 & -0.01 & -0.21 * \\ \text { Extracción de leña } & +0.04 & -0.09 & -0.31 * * & +0.00 & -0.23 * \\ \text { Exposición } & -0.10 & -0.08 & +0.21 * & +0.02 & -0.24 * \\ \text { Pastoreo } & +0.11 & +0.17 & -0.19 & -0.01 & +0.12 \\ \text { Pendiente } & +0.03 & +0.02 & +0.00 & -0.02 & +0.03\end{array}$

para ninguna de las especies. Quercus sebifera no mostró asociaciones estadísticamente significativas con las variables estudiadas (Cuadro 3, Fig. 2).

El DCCA mostró que solamente el primer eje presentó un eigenvalue (Ter Braak 1988) muy alto (0.738), mientras que el segundo eje alcanzó una correlación de 0.171 . La correlación especie-ambiente para el primer eje también fue alta $(\mathrm{r}=0.857)$ y para el segundo fue de 0.518 (Cuadro 4).

La suma de las varianzas de los ejes uno y dos (del DCCA) es de $29 \%$ en la composición de las especies del subgénero Lepidobalanus (Cuadro 4). El primer eje explica un porcentaje relativamente alto de la varianza acumulada de la relación especie-ambiente, la cual mide el

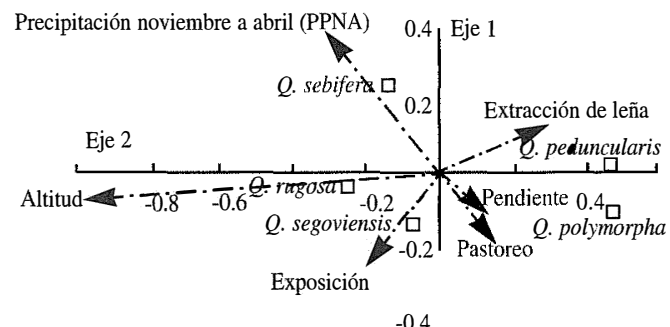

Fig. 2. Diagrama de los primeros dos ejes generados en un análisis de DCCA. Se representan las especies Quercus peduncularis, $Q$. polymorpha, $Q$. rugosa, $Q$. sebifera y $Q$. segoviensis (puntos) y las seis variables ambientales incluidas en el análisis (flechas). La escala de los ejes de las especies está reducida en una tercera parte. Solamente se incluyeron las parcelas cuyas especies tuvieron un ID $\geq 3$. grado de relación entre las especies y el ambiente (Cuadro 4). Se encontró correlación entre la variación de los ID $\geq 3$ de estas especies y las variables ambientales (prueba de permutación de Monte Carlo, $\mathrm{F}=15.04$, p<0.01).

El eje uno explicó un $23.6 \%$ de la variación en la distribución de estas especies y puede ser considerado para representar un gradiente de altitud, aunque también tuvieron una correlación significativa con este eje las variables extracción de leña y exposición. Los más importantes componentes del eje dos de ordenación fueron la PPNA y la altitud, sin embargo, también las variables pastoreo, extracción de leña y exposición $(\mathrm{p}<0.05)$ correlacionaron significativamente con este eje (Cuadro 4 y Fig. 2).

Las variables ambientales más importantes que determinan la distribución de Quercus peduncularis, $Q$. polymorpha, $Q$. rugosa, $Q$. sebifera y $Q$. segoviensis son para el eje uno la altitud y para el segundo la PPNA y la altitud. Estas dos variables pueden definir hábitats diferenciados para estas especies cuando se utilizan las parcelas en las que las especies alcanzaron un valor de ID $\geq 3$; siendo la altitud la que mejor separa los hábitats (Fig. 3).

\section{DISCUSIÓN}

La altitud resultó ser la variable ambiental determinante para separar el hábitat de las especies del género Quercus en la Meseta Central de Chiapas (Figs. 2 y 3). Soto Pinto (1982) y Valencia Ávalos (1989) encontraron 


\section{CUADRO 4}

Eigenvalues, correlaciones especie-ambiente, porcentaje de la varianza explicada usando DCCA y coeficientes de correlación

\begin{tabular}{llllc}
\multicolumn{1}{c}{ Ejes } & \multicolumn{1}{c}{1} & \multicolumn{1}{c}{3} & 4 \\
Eigen valores & 0.738 & 0.171 & 0.03 & 0.010 \\
Correlaciones especie-ambiente & 0.857 & 0.518 & 0.229 & 0.102 \\
$\begin{array}{l}\text { Varianza porcentual acumulada } \\
\text { de los datos de las especies }\end{array}$ & 23.6 & & 30.0 & 30.3 \\
de la relación especie-ambiente & 71.3 & 29.0 & 90.7 & 91.6 \\
Coeficientes de correlación & & 87.7 & \\
Altitud & $-0.6902^{* * *}$ & $-0.3354^{* *}$ & \\
Precipitación nov-abril (PPNA) & -0.1751 & $-0.3971^{* *}$ & \\
Extracción de leña & $+0.2985^{*}$ & $+0.2490^{*}$ & \\
Exposición & $-0.2356^{*}$ & $-0.2555^{*}$ & \\
Pastoreo & -0.1125 & $+0.2854^{*}$ & \\
Pendiente & -0.0349 & +0.0258 &
\end{tabular}

${ }^{*} \mathrm{p}<0.05,{ }^{* *} \mathrm{p}<0.01,{ }^{* * *} \mathrm{p}<0.001$

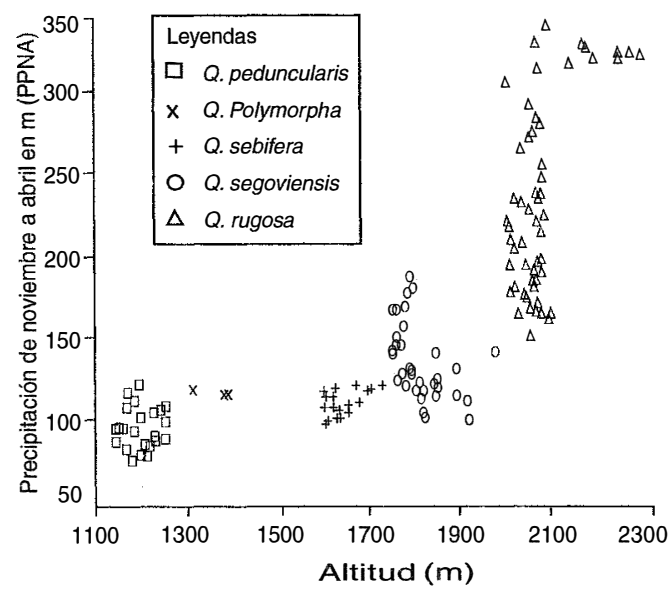

Fig. 3. Distribución de Quercus peduncularis, $Q$. polymorpha, $Q$. rugosa, $Q$. sebifera y $Q$. segoviensis en función de la altitud y la precipitación invernal en la Meseta Central de Chiapas. Únicamente se incluyen las parcelas donde las especies alcanzaron un ID $\geq 3$.

un patrón similar de separación ecológica entre las especies del subgénero Lepidobalanus presentes en Guerrero (Quercus corrugata, $Q$. peduncularis y $Q$. rugosa). Aunque estos dos trabajos se basaron en observaciones de presencia, sin considerar ni abundancia ni cobertura de las especies y sin análisis estadístico.

En la Meseta Central de Chiapas la precipitación anual varía de 1030 a 1975 mm y el periodo de mayor cantidad de lluvias se presenta en los meses de mayo a octubre. De noviembre a abril la precipitación es diferente entre los tipos climáticos, así, el subcálido presenta el $7 \%$ de la precipitación total durante este periodo, el subtemplado el 9\%, el subtemplado húmedo el $11 \%$ y el templado el $16 \%$ de la precipitación total (García 1987). Este periodo de menor precipitación es el que ejerce mayor influencia sobre los periodos fenológicos de las especies vegetales. De las especies estudiadas, se encontró que Quercus peduncularis, $Q$. polymorpha, $Q$. segoviensis y $Q$. sebifera pierden sus hojas durante la estación seca, estrategia desarrollada como respuesta al estrés hídrico (Madrigal Jiménez 1996-1997). Conforme a los resultados encontrados, las tres primeras especies mostraron correlaciones significativas con la variable PPNA. Los sitios donde $Q$. peduncularis y $Q$. segoviensis presentaron ID $\geq 3$, correspondieron a áreas con baja PPNA.

En la región de estudio, las áreas con menor pendiente se han dedicado a actividades agrícolas y ganaderas desde hace siglos (Collier 1975), por lo que las áreas boscosas han quedado restringidas a sitios de mayor pendiente, por ello posiblemente esta variable no resultó significativa. De hecho, todas las parcelas estudiadas se encontraron en pendientes mayores a seis grados. 
Para que el pastoreo alcanzara una correlación estadísticamente significativa, se requería de un valor mínimo de 0.20 , el que ninguna especie a canzó. Sin embargo el valor de la correlación con Quercus rugosa fue de 0.19 (Cuadro 3), lo que sugiere que probablemente la actividad ganadera afecte en forma negativa la distribución de esta especie. Ramírez-Marcial et al. (1996) encontraron que el apacentamiento del ganado reduce la oportunidad de establecimiento de semillas y plántulas de Quercus. De hecho, los ovinos ramonean los rebrotes de encino en períodos de escasez hídrica (Nahed Toral y Parra Vázquez 1984).

El presente estudio sugiere que Quercus peduncularis, $Q$. polymorpha, $Q$. rugosa, $Q$. sebifera y $Q$. segoviensis presentan hábitat diferenciados y que estos están determinados por variables naturales, en este caso la altitud y PPNA fueron las determinantes (Fig. 3). Lo anterior coincide con Van Valen (1976) en el sentido de que es posible utilizar el concepto de especie ecológica con el manejo de una zona adaptativa que delimite a las especies. Las variables culturales como extracción de leña y pastoreo, también condicionan la distribución y abundancia de estas especies.

En un programa de manejo y restauración de bosques es recomendable considerar las variables naturales y culturales para delimitar áreas favorables para especies de interés productivo, como en este caso las especies de Quercus. La altitud y la PPNA permiten estratificar a la Meseta Central de Chiapas y plantear para cada estrato la o las especies mejor adaptadas. La información que genera este trabajo proporciona información básica acerca de la ecología de las especies del género Quercus con lo que facilita su utilización en programas de reforestación, restauración y manejo, suministra información precisa de las áreas donde hay poco traslape entre las especies, asegurando la recolección de semilla con menor probabilidad de mezclas interespecíficas y señala las áreas donde estas especies se pueden establecer con mayor posibilidad de éxito.

\section{AGRADECIMIENTOS}

Agradecemos a D. Golicher y N. Ramírez Marcial por su asesoría en el análisis estadístico. Tres revisores anónimos aportaron valiosos comentarios. S. Valencia Ávalos, G. Domínguez Vázquez y M. Martínez Icó realizaron la identificación botánica. D. Méndez Ton apoyó con la localización geográfica de las especies. I. March Mifsut facilitó el acceso al Laboratorio de Análisis Geográfico (LAIGE-ECOSUR). El Consejo Nacional de Ciencia y Tecnología otorgó beca para J.G. Alvarez Moctezuma (\# 95538) y apoyó con recursos a través del proyecto \# 2140-N9303. El ECOSUR proporcionó la infraestructura y subsidio para el desarrollo de este trabajo. Fondos recurrentes provinieron de US-Environmental Protection Agency (US-EPA) bajo convenio con ECOSUR (CR822200). La UNICACH proporcionó apoyo logístico.

\section{RESUMEN}

El objetivo de este trabajo fue describir el hábitat de las especies del subgénero Lepidobalanus en la Meseta Central de Chiapas e identificar las principales variables que condicionan su distribución. En 258 parcelas se utilizó un índice de dominancia, basado en la abundancia y cobertura de las especies, para conocer la distribución de Quercus peduncularis, $Q$. polynorpha, $Q$. rugosa, $Q$. sebifera y $Q$. segoviensis. Además se midieron las variables ambientales: altitud, precipitación de noviembre a abril (PPNA), exposición, pendiente, intensidad de extracción de leña y pastoreo. Se utilizó el Detrended Canonical Correspondence Analysis para la evaluación estadística de los datos. Se encontró una relación negativa entre el índice de dominancia de $Q$. peduncularis y $Q$. segoviensis con la altitud y la PPNA. El índice de dominancia de $Q$. rugosa y Q. segoviensis mostró correlación con la exposición y la intensidad de extracción de leña. La altitud fue la variable ambiental que condiciona la distribución diferencial de las especies, seguida por la PPNA. El resultado de este estudio sugiere que hay hábitat diferenciados para $Q$. peduncularis, $Q$. polymorpha, $Q$. rugosa, $Q$. sebifera y $Q$. segoviensis determinados por variables naturales (altitud y PPNA), aunque también las variables culturales (pastoreo y extracción de leña) pueden condicionar la distribución y abundancia de estas especies. 


\section{REFERENCIAS}

Anónimo. 1974. Carta topográfica. Escala 1:50 000. Instituto Nacional de Estadística, Geografía e Informática. Secretaría de Programación y Presupuesto. México, D.F.

Anónimo. 1984. Carta de efectos climáticos regionales Noviembre-Abril. Escala 1:250000. Instituto Nacional de Estadística, Geografía e Informática. Secretaría de Programación y Presupuesto. México, D.F.

Berlin, B., D.E. Breedlove \& P.H. Raven. 1974. Principles of Tzeltal Plant Classification: An Introduction to the Botanical Ethnography of a Mayan-Speaking People of Highland Chiapas. Academic, Londres. 660 p.

Braun-Blanquet, J. 1979. Fitosociología. Bases para el Estudio de las Comunidades Vegetales. Blume, Madrid. 820 p.

Breedlove, D.E. 1981. Introduction to the flora of Chiapas. Flora of Chiapas. 1:1-35.

Breedlove, D.E. \& R.M. Laughlin. 1993. The Flowering of Man: A Tzotzil Botany of Zinacantán. Smithsonian Institution, Washington D.C. 706 p.

Buschbacher, R.J. 1990. Natural forest management in the humid tropics: considerations. Ambio 19: 253-8.

Collier, G.A. 1975. Fields of the Tzotzil: Ecological Bases of Tradition in Highland Chiapas. Universidad de Texas. Austin, Texas. 255 p.

Díaz Hernández, B.M., G. Jiménez Ferrer, D. Ramírez Ávila, J.M. Comparán Rodríguez y M. Aguilar López. 1988. Energía Rural en México. Análisis de la Estructura del Consumo de la Macroregión Pacífico Sur, México. Secretaría de Energía, Minas e Industria Paraestatal y Comisión de la Comunidad Europea. México. 70 p.

García, E. 1987. Modificaciones al Sistema de Clasificación Climática de Köppen para Adaptarlo a las Condiciones de la República Mexicana. Instituto de Geografía. Universidad Nacional Autónoma de México. México, D.F. 246 p.

González-Espinosa, M., S. Ochoa-Gaona, N. RamírezMarcial \& P. F. Quintana-Ascencio. 1995. Current land-use trends and conservation of old-growth forest habitats in the highlands of Chiapas, Mexico, p. 190198. In M. Wilson, S. Sader \& A. Estrada (eds.). Conservation of neotropical migrant birds in Mexico. Mexico-US-Canada. Maine Agriculture and Forest Experiment Station. Miscellaneous Publication 727.

González-Espinosa, M., S. Ochoa-Gaona, N. RamírezMarcial \& P.F. Quintana-Ascencio. 1997. Contexto vegetacional y florístico de la agricultura en los Altos de Chiapas, p. 91-124. In M.R. Parra Vázquez \& B.M. Díaz Hernández (eds.). Los Altos de Chiapas: agricultura y crisis rural, Tomo I: Los Recursos Naturales. El Colegio de la Frontera Sur. San Cristóbal de Las Casas, Chiapas, México.

Hill, M.O. \& H.G. Gauch Jr. 1980. Detrended correspondence analysis: an improved ordination technique. Vegetatio 42: 47-58.

Madrigal Jiménez, T.A. 1996-1997. Fenología y ecofisiología del Quercus oocarpa (Fagaceae), Cartago, Costa Rica. Rev. Biol. Trop. 44/45: 117-123.

Montoya Gómez, G. 1995. La explotación maderera en la subregión San Cristóbal y las reformas en el artículo 27 constitucional, p. 33-46. In Miranda Ocampo, R. (comp.). Chiapas el regreso a la utopía. Comuna. Universidad Autónoma de Guerrero. Chilpancingo, Guerrero, México.

Müllerried, F.K.G. 1957. Geología de Chiapas. Publicaciones del Gobierno del Estado de Chiapas. Tuxtla Gutiérrez, Chiapas, México. 180 p.

Nahed Toral, J. \& M.R. Parra Vázquez. 1984. Ovinocultura en Los Altos de Chiapas: un sistema tradicional. Rev. Mex. Prod. Animal. 6: 25-41.

Quintana-Ascencio, P.F. \& M. González-Espinosa. 1993. Afinidad fitogeográfica y papel sucesional de la flora leñosa de los bosques de pino-encino de Los Altos de Chiapas, México. Acta Bot. Mex. 21:43-57.

Ramírez-Marcial, N., M. González-Espinosa \& E. GarcíaMoya. 1996. Establecimiento de Pinus spp. y Quercus spp. en matorrales y pastizales de Los Altos de Chiapas, México: Agrociencia 30: 249-57.

Roberts, D.W. 1986. Ordination on the basis of fuzzy set theory. Vegetatio 66: 123-31.

Soto Pinto, M.L. 1982. Estudio taxonómico del género Quercus (Fagaceae) de la cuenca del Río Zopilote, Guerrero. Tesis de Biología. Universidad Nacional Autónoma de México. México, D.F. 141 p.

Ter Braak, C.J.F. 1988. Canonical correspondence analysis: a new eigenvector technique for multivariate direct gradient analysis. Ecology 67: 1167-79.

Valencia Ávalos, S. 1989. Contribución al conocimiento del género Quercus (Fagaceae) en el estado de Guerrero, México. Tesis de Biología. Universidad Nacional Autónoma de México. México, D.F. 183 p.

Van Valen, L. 1976. Ecological species, multispecies, and oaks. Taxon 25: 233-9.

Vickery, J.A., W.J. Sutherland, M. O'Brien, A.R. Watkinson \& A. Yallop. 1997. Managing coastal grazing marshes for breeding waders and overwintering geese: is there a conflict? Biol. Conserv. 79: 23-34. 